\title{
A Grey Interval Relational Degree-Based Dynamic Multiattribute Decision Making Method and Its Application in Investment Decision Making
}

\author{
Yuhong Wang, ${ }^{1}$ Xiaojuan Shi, ${ }^{1}$ Jihong Sun, ${ }^{2}$ and Wuyong Qian ${ }^{1}$ \\ ${ }^{1}$ School of Business, Jiangnan University, Wuxi, Jiangsu 214122, China \\ ${ }^{2}$ National Institute of Education Sciences, Beijing 100088, China \\ Correspondence should be addressed to Yuhong Wang; wyh2003@gmail.com
}

Received 25 August 2013; Accepted 17 March 2014; Published 14 April 2014

Academic Editor: Jian Guo Zhou

Copyright ( $\odot 2014$ Yuhong Wang et al. This is an open access article distributed under the Creative Commons Attribution License, which permits unrestricted use, distribution, and reproduction in any medium, provided the original work is properly cited.

The purpose of this paper is to propose a three-dimensional grey interval relational degree model for dynamic Multiattribute decision making. In the model, the observed values are interval grey numbers. Elements are selected in the system as the points in an $m$-dimensional linear space. Then observation data of each element to different time and objects are as the coordinates of point. An optimization model is employed to obtain each scheme's affiliate degree for the positive and negative ideal schemes. And a three-dimensional grey interval relational degree model based on time, index, and scheme is constructed in the paper. The result shows that the three-dimensional grey relational degree simplifies the traditional dynamic multiattribute decision making method and can better resolve the dynamic multiattribute decision making problem of interval numbers. The example illustrates that the method presented in the paper can be used to deal with problems of uncertainty such as dynamic multiattribute decision making.

\section{Introduction}

Grey relational analysis (GRA) is one of the main contents of grey system theory [1], which is an analytical method based on grey relation topological space. Its basic idea is to judge the interfactor relation degree according to the proximity and similarity degree of the order change between curves. As we all know, the method of grey relation degree does not impose high requirements for the size of samples and does not need typical distribution rules. And computational amount is small. Results of qualitative and quantitative analysis can be matched well, which can be widely applied in the decision making fields $[2,3]$.

In the social and economic systems, there exist a large number of comprehensive decision making problems of complicated system influenced by dynamic multiattribute indexes. The basic features of such kind of problems are to add the time space on the basis of decision space and target space, which are three-dimension decision sequential problems of time, index, and scheme. As for such problems, we can not directly use the method of grey relation degree. Fan and Xiao [4] combined the ideal point method of multiattribute decision making with the extended grey relation degree applying the GRA to the dynamic multiattribute decision making for the first time. Yang [5] utilized the square and minimum of relational dissimilarity degree to ascertain the affiliate degree and improved the resolution of ordering results. Zeng and Luo [6] gave three kinds of relational coefficients and selected different types of relational coefficients according to features of different indexes, then gave results of decision sorting and total sequencing in each time quantum. However, this method has strong subjective elements, and ordering results are different from those of Fan and Xiao [4]. Wang [7] took development character into consideration and utilized the "rewards and punishment" principle to a preliminary process of indexes and constructed an optimized index system to ensure that the weight of each index is in the optimized index system. Owing to the difference of index weights, the ordering result is also different from Fan and Xiao [4]. Dang et al. [8] used the vague concept and the theoretical ideas of set pair 
analysis to propose a linear transformation operator $[-1,1]$, which is easy to be calculated. This method is quite practical and could be used to seek a negative/positive ideal scheme in each time quantum and it established a decision making model based on the dynamic multiattribute GRA.

The existing interval number decision-making models [9-13] separate the interval number decision matrix into upper-bound and lower-bound matrixes. For the upperbound and lower-bound matrixes, their relations with the virtual positive ideal scheme and negative ideal scheme could be used to set up optimized decision model. By doing this, the original interval number is separated purposefully and which makes the result not match reality. According to the theory of grey relational degree, the nearer the geometric distance of the sequence distance is, the bigger the grey relational degrees are. A grey interval relational model based on three dimensions of time, index, and scheme is constructed. Combining with ideal point method of the multiattribute decision making, through the computation of interval number relational degree in order to set up an optimized affiliate degree model, this paper tries to seek the best decision scheme of the dynamic multiattribute decision problem with interval numbers. The remainder of this paper is organized as follows. In Section 2, the methods for standardization of the interval number decision matrix are presented. Section 3 presents the method of three-dimensional grey interval relational degrees. Section 4 discusses the dynamic multiattribute decision making method of interval number based on three-dimensional grey interval relation degree. An example analysis is presented in Section 5. Conclusions are drawn in Section 6.

\section{Methods for Standardization of an Interval Number Decision Matrix}

Definition 1. Assume that a multiattribute decision making problem has $n$ evaluation objects or formulated decision schemes to form the scheme set $S, S=\left\{S_{1}, S_{2}, \ldots, S_{n}\right\} ; m$ evaluation indexes or attributes form an index set $A, A=$ $\left\{A_{1}, A_{2}, \ldots, A_{m}\right\}$; the attribute value of scheme $S_{i}$ for index $A_{j}$ is $\left[x_{i j}^{L}, x_{i j}^{U}\right](i=1,2, \ldots, n ; j=1,2, \ldots, m)$. So the interval number decision making matrix of scheme set $S$ for index set $A$ is

$$
X=\left[\begin{array}{cccc}
{\left[x_{11}^{L}, x_{11}^{U}\right]} & {\left[x_{12}^{L}, x_{12}^{U}\right]} & \cdots & {\left[x_{1 m}^{L}, x_{1 m}^{U}\right]} \\
{\left[x_{21}^{L}, x_{21}^{U}\right]} & {\left[x_{22}^{L}, x_{22}^{U}\right]} & \cdots & {\left[x_{2 m}^{L}, x_{2 m}^{U}\right]} \\
\cdots & \cdots & \cdots & \cdots \\
{\left[x_{n 1}^{L}, x_{n 1}^{U}\right]} & {\left[x_{n 2}^{L}, x_{n 2}^{U}\right]} & \cdots & {\left[x_{n m}^{L}, x_{n m}^{U}\right]}
\end{array}\right]
$$

The index attribute set $A=\left\{A_{1}, A_{2}, \ldots, A_{m}\right\}$ generally can be divided into two types, namely, "beneficial type" and "cost type". The "beneficial type" index means that the larger the value, the better the value; while the "cost type" index means that the smaller the value, the better the value.

Owing to the fact that the centralized indexes have different dimensions, when making a decision, it is hard to compare them directly. Then we need to have a normalization process with the original decision making matrix.

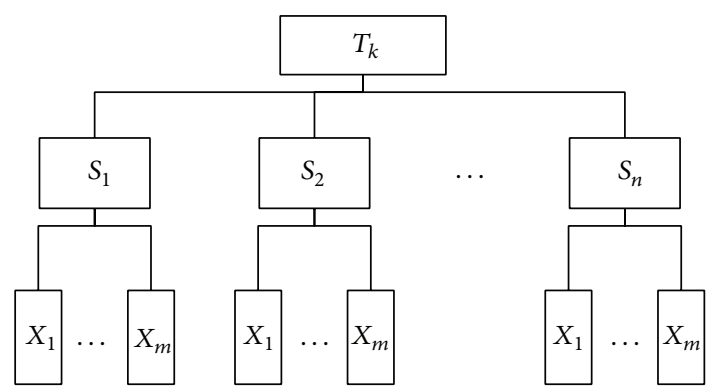

FIGURE 1: The structure of a three-dimensional decision making system.

If $A_{j}$ is a beneficial type index, then

$$
r_{i j}^{L}=\frac{x_{i j}^{L}}{\sum_{i=1}^{n} x_{i j}^{U}}, \quad r_{i j}^{U}=\frac{x_{i j}^{U}}{\sum_{i=1}^{n} x_{i j}^{L}} .
$$

If $A_{j}$ is a cost type index, then

$$
r_{i j}^{L}=\frac{1 / x_{i j}^{U}}{\sum_{i=1}^{n}\left(1 / x_{i j}^{L}\right)}, \quad r_{i j}^{U}=\frac{1 / x_{i j}^{L}}{\sum_{i=1}^{n}\left(1 / x_{i j}^{U}\right)} .
$$

This normalization method makes the normalization transformation of the decision matrix $X$ get the normalization decision making matrix $R$ :

$$
\begin{aligned}
R & =\left[\begin{array}{cccc}
{\left[r_{11}^{L}, r_{11}^{U}\right]} & {\left[r_{12}^{L}, r_{12}^{U}\right]} & \cdots & {\left[r_{1 m}^{L}, r_{1 m}^{U}\right]} \\
{\left[r_{21}^{L}, r_{21}^{U}\right]} & {\left[r_{22}^{L}, r_{22}^{U}\right]} & \cdots & {\left[r_{2 m}^{L}, r_{2 m}^{U}\right]} \\
\ldots & \cdots & \cdots & \cdots \\
{\left[r_{n 1}^{L}, x_{n 1}^{U}\right]} & {\left[r_{n 2}^{L}, r_{n 2}^{U}\right]} & \cdots & {\left[r_{n m}^{L}, r_{n m}^{U}\right]}
\end{array}\right] \\
& =\left[r_{1}, r_{2}, \ldots, r_{m}\right],
\end{aligned}
$$

where $r_{i}=\left(\left[r_{i 1}^{L}, r_{i 1}^{U}\right],\left[r_{i 2}^{L}, r_{i 2}^{U}\right], \ldots,\left[r_{i m}^{L}, r_{i m}^{U}\right]\right)(i=1,2, \ldots, n)$ is the interval number effect vector for scheme $i$. Obviously, the normalization decision matrix $R$ 's elements are $r_{i j}^{L}, r_{i j}^{U} \in$ $[0,1](i=1,2, \ldots, n ; j=1,2, \ldots, m)$.

\section{Three-Dimensional Grey Interval Relational Degree}

Assume that the sample point of a three-dimensional decision making system consists of time $T_{k}(k=1,2, \ldots, t)$, index $X_{i}(i=1,2, \ldots, m)$, and scheme $S_{j}(j=1,2, \ldots, n)$. Thus the structure of a three-dimensional decision making system can be described as Figure 1 .

A grey relational space is composed of a distance space and a topological space. The "distance" in the distance space is a measure in multiple comparisons, while the point set topology is a neighborhood and complete comparison. Then the combination of the two constructs the complete comparison with measurement, which is also the basic feature of grey relational decision making. We will take each element in the system as the point in the $m$-dimensional linear space and take its observation data of different time and objects 
as the coordinates of the point. And a calculation formula of grey interval relational degree which is suitable for the three-dimensional decision making system is presented in the paper.

Assume $\tilde{a}=\left[a^{L}, a^{U}\right],\left(a^{L} \leq a^{U}\right), \tilde{a}$ is an interval number. In particular, if $a^{L}=a^{U}$, then $\tilde{a}$ degenerates into a real number.

Definition 2. Assume that sequences of $m$-dimensional interval numbers are $A=\left\{\left(a_{1}^{L}, a_{1}^{U}\right),\left(a_{2}^{L}, a_{2}^{U}\right), \ldots,\left(a_{m}^{L}, a_{m}^{U}\right)\right\}, a_{i}^{L} \leq$ $a_{i}^{U} ; B=\left\{\left(b_{1}^{L}, b_{1}^{U}\right),\left(b_{2}^{L}, b_{2}^{U}\right), \ldots,\left(b_{m}^{L}, b_{m}^{U}\right)\right\}, b_{i}^{L} \leq b_{i}^{U}, i=$ $1,2, \ldots, m$; namely,

$$
\begin{aligned}
& d_{p}(A, B) \\
& =2^{-1 / p}\left[\left(a_{1}^{L}-b_{1}^{L}\right)^{p}+\left(a_{1}^{U}-b_{1}^{U}\right)^{p}+\cdots\right. \\
& \left.\quad+\left(a_{m}^{L}-b_{m}^{L}\right)^{p}+\left(a_{m}^{U}-b_{m}^{U}\right)^{p}\right]^{1 / p}
\end{aligned}
$$

is the distance between sequence $A$ and sequence $B$ of $m$ dimensional interval numbers. When $p=2$, then $d_{2}(A, B)$ is an Euclidean distance.

When $A$ and $B$ are real number sequences, that is, $a_{i}^{L}=$ $a_{i}^{U}, b_{i}^{L}=b_{i}^{U}, i=1,2, \ldots, m, d_{2}(A, B)$ represents the Euclidean distance between real numbers $A$ and $B$. So the distance of $m$-dimensional interval number sequence is the extension of the distance of $m$-dimensional real number sequences.

Definition 3. Assume that the system behavior sequence at time $k$ is

$$
\begin{array}{r}
\widetilde{X}_{i}^{k}=\left\{\widetilde{x}_{i}^{k}(1), \widetilde{x}_{i}^{k}(2), \ldots, \widetilde{x}_{i}^{k}(m)\right\}, \\
\text { where } i=0,1,2, \ldots, n .
\end{array}
$$

Let the obscure set include core $I[a: \odot \vdots b]$ and become the grey area of sequence set $\widetilde{X}=\left\{\widetilde{X}_{i}, i=0,1, \ldots, n\right\}$. And the interval $[a, b]$ is called the environment of sequence $\widetilde{X}_{i} \cdot a$ and $b$ are the low-environment parameter and up-environment parameter of sequence $\widetilde{X}_{i}$, respectively. Environment parameters $a$ and $b$ express the completeness of the system, and generally $a=\min _{i} d\left(X_{0}, X_{i}\right), b=\max _{i} d\left(X_{0}, X_{i}\right)$, which makes the relational degree of the two sequences not only depend on themselves but also depend on other element sequences. Sequence $X_{i}$ is an effect measurement (EM) after the grey generation, which has uniform polarities.

Assume that $X_{i}^{k}(i=0,1, \ldots, n)$ is regarded as the $n+1$ points in the $m$-dimensional linear space; each element's data observation of different time and objects are regarded as coordinates. And the interrelations among elements are studied in the specific $m$-dimensional linear space. An improved grey relational degree model which is similar to Deng's grey relational degree model is constructed.
Definition 4. For $\xi \in(0,1)$, assume that

$$
\begin{gathered}
\xi\left(X_{0}^{k}, X_{i}^{k}\right) \\
=\frac{\min _{i} \min _{k} d_{p}\left(X_{0}^{k}, X_{i}^{k}\right)+\rho \max _{i} \max _{k} d_{p}\left(X_{0}^{k}, X_{i}^{k}\right)}{d_{p}\left(X_{0}^{k}, X_{i}^{k}\right)+\rho \max _{i} \max _{k} d_{p}\left(X_{0}^{k}, X_{i}^{k}\right)}, \\
\gamma\left(X_{0}, X_{i}\right)=\sum_{k=1}^{t} \lambda_{k} \xi\left(X_{0}^{k}, X_{i}^{k}\right),
\end{gathered}
$$

where $0<\lambda_{k}<1, \sum_{k=1}^{t} \lambda_{k}=1, \rho$ is the resolution coefficient, generally $\rho=0.5, d_{p}\left(X_{0}, X_{i}\right)$ is a distance between system behavior feature sequence $X_{0}$ and element sequence $X_{i}$.

Then the expression $\gamma\left(X_{0}, X_{i}\right)$ is called the threedimensional grey interval relational degree. Obviously, threedimensional grey interval relational degree $\gamma\left(X_{0}, X_{i}\right)$ satisfies grey relational four axioms.

\section{The Dynamic Multiattribute Decision Making Method of Interval Number Based on Three-Dimensional Grey Interval Relational Degree}

Assume that the time quantum of the dynamic multiattribute decision making problem is $T_{k}(k=1,2, \ldots, t)$, the corresponding weight is $\lambda_{k}\left(0<\lambda_{k}<1, \sum_{k=1}^{t} \lambda_{k}=1\right)$, the index is $X_{j}(j=0,1, \ldots, n)$, the corresponding weight is $w_{j}\left(0<w_{j}<1, \sum_{j=1}^{n} w_{j}=1\right)$, and the decision making scheme is $S_{i}(i=1,2, \ldots, m) . S_{i}$ is assumed as a noninferior solution. There are a lot of methods such as Delphi method and Analytic Hierarchy Process to obtain weight $\lambda_{k}$ and $w_{j}$ in practical applications.

Elements in the interval number decision-making matrix have been changed by a normalization process. This process could lead to consistent changes for "benefit type" and "cost type" indexes in the matrix. The bigger the index is, the better the scheme is after the interval numbers through a normalization process. So when we construct the optimized scheme, we should choose the scheme which has the largest corresponding interval number in the interval number normalization decision matrix as the best scheme. And we could use a mean number of the upper and lower bounds of an interval number as the specific number value. We can construct the best scheme selection method for a multiattribute decision making problem in the following.

Definition 5. Assume that

$$
\begin{array}{r}
r_{i_{0}^{+} j}^{k}=\max \left\{\frac{r_{i j}^{L k}+r_{i j}^{U k}}{2} \mid 1 \leq i \leq n\right\} \\
(j=1,2, \ldots, m ; k=1,2, \ldots, t),
\end{array}
$$

and thus

$$
S_{0+}^{k}=\left\{\left[r_{i_{0}^{+} 1}^{L k}, r_{i_{0}^{+} 1}^{U k}\right],\left[r_{i_{0}^{+} 2}^{L k}, r_{i_{0}^{+} 2}^{U k}\right], \ldots,\left[r_{i_{0}^{+} m}^{L k}, r_{i_{0}^{+} m}^{U k}\right]\right\}
$$

is believed to be the positive ideal scheme. 
Definition 6. Assume that

$$
\begin{aligned}
r_{i_{0}^{-} j}^{k}= & \min \left\{\frac{\left(r_{i j}^{L k}+r_{i j}^{U k}\right)}{2 \mid 1 \leq i \leq n}\right\} \\
& (j=1,2, \ldots, m ; k=1,2, \ldots, t),
\end{aligned}
$$

and thus

$$
S_{0-}^{k}=\left\{\left[r_{i_{0}^{-} 1}^{L k}, r_{i_{0}^{-1} 1}^{U k}\right],\left[r_{i_{0}^{-2}}^{L k}, r_{i_{0}^{-} 2}^{U k}\right], \ldots,\left[r_{i_{0}^{-} m}^{L k}, r_{i_{0}^{-} m}^{U k}\right]\right\}
$$

is believed to be the negative ideal scheme.

Assume that, through the Delphi method and AHP method, the corresponding weight vector of each time quantum is

$$
\begin{gathered}
\lambda=\left(\lambda_{1}, \lambda_{2}, \ldots, \lambda_{t}\right), \quad \lambda_{k}>0 \quad(k=1,2, \ldots, t), \\
\sum_{k=1}^{t} \lambda_{k}=1 .
\end{gathered}
$$

Each index's weigh vector is

$$
\begin{gathered}
w=\left(w_{1}, w_{2}, \ldots, w_{m}\right), \quad w_{j}>0 \quad(j=1,2, \ldots, m), \\
\sum_{j=1}^{m} w_{j}=1 .
\end{gathered}
$$

The relational coefficient of the $i$ scheme and the positive ideal scheme $S_{0+}^{k}$ is

$$
\begin{array}{r}
\xi^{+}\left(S_{0+}^{k}, X_{i}^{k}\right)=\frac{\min _{i} \min _{k} d_{p}\left(S_{0+}^{k}, X_{i}^{k}\right)+\rho \max _{i} \max _{k} d_{p}\left(S_{0+}^{k}, X_{i}^{k}\right)}{d_{p}\left(S_{0+}^{k}, X_{i}^{k}\right)+\rho \max _{i} \max _{k} d_{p}\left(S_{0+}^{k}, X_{i}^{k}\right)} \\
(i=1,2, \ldots, n ; k=1,2, \ldots, t) .
\end{array}
$$

The relational coefficient of the $i$ scheme and the negative ideal scheme $S_{0-}^{k}$ is

$$
\begin{array}{r}
\xi^{-}\left(S_{0-}^{k}, X_{i}^{k}\right)=\frac{\min _{i} \min _{k} d_{p}\left(S_{0-}^{k}, X_{i}^{k}\right)+\rho \max _{i} \max _{k} d_{p}\left(S_{0-}^{k}, X_{i}^{k}\right)}{d_{p}\left(S_{0-}^{k}, X_{i}^{k}\right)+\rho \max _{i} \max _{k} d_{p}\left(S_{0-}^{k}, X_{i}^{k}\right)} \\
(i=1,2, \ldots, n ; k=1,2, \ldots, t) .
\end{array}
$$

The three-dimensional grey interval number relational degrees of the $i$ scheme for positive/negative ideal schemes are below:

$$
\begin{gathered}
\gamma^{+}\left(S_{0+}, X_{i}\right)=\sum_{k=1}^{t} \lambda_{k} \xi^{+}\left(S_{0+}^{k}, X_{i}^{k}\right) \\
\gamma^{-}\left(S_{0-}, X_{i}\right)=\sum_{k=1}^{t} \lambda_{k} \xi^{-}\left(S_{0-}^{k}, X_{i}^{k}\right) .
\end{gathered}
$$

Obviously, the bigger $\gamma^{+}\left(S_{0+}, X_{i}\right)$ is, the closer between decision making scheme $S_{i}$ and positive ideal scheme $S^{+}$is; then the significance of $\gamma^{-}\left(S_{0-}, X_{i}\right)$ goes the opposite way and is lower. So the best decision making scheme should have the highest relational degree with the positive ideal scheme while having the lowest relational degree with the negative ideal scheme. Assume that the affiliate values of $i$ th scheme $S_{i}$ for positive/negative ideal schemes are $\mu_{i}$ and $1-\mu_{i}$, so we get the objective function

$$
\begin{aligned}
\min F(\mu)= & \sum_{i=1}^{n}\left[\left(1-\mu_{i}\right) \gamma^{+}\left(S_{0+}, X_{i}\right)\right]^{2} \\
& +\sum_{i=1}^{n}\left[\mu_{i} \gamma^{-}\left(S_{0-}, X_{i}\right)\right]^{2}
\end{aligned}
$$

and then we have

$$
\mu_{i}=\frac{\left[\gamma^{+}\left(S_{0+}, X_{i}\right)\right]^{2}}{\left[\gamma^{+}\left(S_{0+}, X_{i}\right)\right]^{2}+\left[\gamma^{-}\left(S_{0-}, X_{i}\right)\right]^{2}} .
$$

According to magnitude of $\mu_{i}$, we arrange all the schemes in sequence. The bigger $\mu_{i}$ is, the closer the evaluated scheme and ideal optimized scheme are, so the decision making scheme $S_{i}$ is better. We should also emphasize that the special cases will occur in the following situation. Only when the distance between the alternative and PIS is equal to the distance between the alternative and NIS, the degree of grey relational coefficient of alternative from PIS is equal to the degree of grey relational coefficient of alternative from NIS.

\section{A Case Analysis}

An investment bank decides to make an investment in three enterprises. The expert team investigates and takes part in the evaluation of the three enterprises' social benefit index, environment pollution (also called benefit index), and economic benefit index from 2010 to 2012. It is convenient to select the focused investment subject according to the comprehensive evaluation of the three indexes. The expert team's evaluation results of each index are in the form of interval numbers (only give an approximate range). We assume that the three enterprises are three schemes $S_{i}(i=1,2,3)$. The interval number decision making matrixes during the three years are below:

$$
\begin{gathered}
X_{1}=\left[\begin{array}{lll}
{[0.35,0.51]} & {[0.30,0.40]} & {[30,40]} \\
{[0.65,0.70]} & {[0.36,0.50]} & {[20,25]} \\
{[0.36,0.60]} & {[0.45,0.65]} & {[20,20]}
\end{array}\right], \\
X_{2}=\left[\begin{array}{lll}
{[0.43,0.58]} & {[0.35,0.45]} & {[26,26]} \\
{[0.43,0.68]} & {[0.50,0.73]} & {[25,30]} \\
{[0.70,0.78]} & {[0.45,0.55]} & {[35,45]}
\end{array}\right], \\
X_{3}=\left[\begin{array}{ccc}
{[0.4,0.61]} & {[0.5,0.55]} & {[40,50]} \\
{[0.7,0.8]} & {[0.4,0.5]} & {[23,28]} \\
{[0.5,0.65]} & {[0.4,0.65]} & {[37,40]}
\end{array}\right] .
\end{gathered}
$$

Step 1. Owing to the fact that the three indexes are all benefit type indexes, we have a normalization process of decision 
making matrixes $X_{1}, X_{2}, X_{3}$ according to (2); then we get normalization interval number decision matrix below:

$$
\begin{aligned}
& R_{1}=\left[\begin{array}{lll}
{[0.1934,0.3750]} & {[0.1935,0.3604]} & {[0.3529,0.5714]} \\
{[0.3591,0.5147]} & {[0.2323,0.4505]} & {[0.2353,0.3571]} \\
{[0.1989,0.4412]} & {[0.2903,0.5856]} & {[0.2353,0.2857]}
\end{array}\right], \\
& R_{2}=\left[\begin{array}{lll}
{[0.2108,0.3718]} & {[0.2023,0.3462]} & {[0.2574,0.3023]} \\
{[0.2108,0.4359]} & {[0.2890,0.5615]} & {[0.2475,0.3488]} \\
{[0.3431,0.5000]} & {[0.2601,0.4231]} & {[0.3465,0.5233]}
\end{array}\right], \\
& R_{3}=\left[\begin{array}{lll}
{[0.1942,0.3813]} & {[0.2941,0.4231]} & {[0.3390,0.5000]} \\
{[0.3398,0.5000]} & {[0.2353,0.3846]} & {[0.1949,0.2800]} \\
{[0.2427,0.4063]} & {[0.2353,0.5000]} & {[0.3136,0.4000]}
\end{array}\right] .
\end{aligned}
$$

Step 2. Construct the positive and negative ideal schemes at each time quantum:

$$
\begin{gathered}
S_{0+}^{1}=\{[0.3591,0.5147],[0.2903,0.5856], \\
[0.3529,0.5714]\} \\
S_{0-}^{1}=\{[0.1934,0.3750],[0.1935,0.3604], \\
[0.2353,0.2857]\} \\
S_{0+}^{2}=\{[0.3431,0.5000],[0.2890,0.5615], \\
\quad[0.3465,0.5233]\} \\
S_{0-}^{2}=\{[0.2108,0.3718],[0.2023,0.3462], \\
\quad[0.2574,0.3023]\} \\
S_{0+}^{3}=\{[0.3398,0.5000],[0.2353,0.5000], \\
\quad[0.3390,0.5000]\} \\
S_{0-}^{3}=\{[0.1942,0.3813],[0.2353,0.3846], \\
[0.1949,0.2800]\} .
\end{gathered}
$$

Step 3. Through Delphi method or AHP method, we make sure of the corresponding weight vectors of each index and time quantum. In order to simplify the analytic process, we assume that $w_{1}=w_{2}=w_{3}=1 / 3$ and $\lambda_{1}=\lambda_{2}=\lambda_{3}=$ $1 / 3$. And we should calculate the interval correlation degree of each scheme to positive/negative ideal scheme according to (14) and (15) (assume $p=2$ ):

$$
\begin{aligned}
\xi^{+}\left(S_{0+}^{1}, X_{1}^{1}\right)= & 0.6409, \quad \xi^{+}\left(S_{0+}^{2}, X_{1}^{2}\right)=0.5813 \\
\xi^{+}\left(S_{0+}^{3}, X_{1}^{3}\right)= & 0.8258 \\
\xi^{+}\left(S_{0+}^{1}, X_{2}^{1}\right)= & 0.6974, \quad \xi^{+}\left(s_{0+}^{2}, X_{2}^{2}\right)=0.7556 \\
& \xi^{+}\left(S_{0+}^{3}, X_{2}^{3}\right)=0.6947 \\
\xi^{+}\left(S_{0+}^{1}, X_{3}^{1}\right)= & 0.6074, \quad \xi^{+}\left(s_{0+}^{2}, X_{3}^{2}\right)=1.0000 \\
\xi^{+}\left(S_{0+}^{3}, X_{3}^{3}\right)= & 0.9210
\end{aligned}
$$

$$
\begin{aligned}
\xi^{-}\left(S_{0-}^{1}, X_{1}^{1}\right)= & 0.3385, \quad \xi^{-}\left(S_{0-}^{2}, X_{1}^{2}\right)=1.0000 \\
& \xi^{-}\left(S_{0-}^{3}, X_{1}^{3}\right)=0.3674 \\
\xi^{-}\left(S_{0-}^{1}, X_{2}^{1}\right)= & 0.3889, \quad \xi^{-}\left(S_{0-}^{2}, X_{2}^{2}\right)=0.3917 \\
& \xi^{-}\left(S_{0-}^{3}, X_{2}^{3}\right)=0.4569 \\
\xi^{-}\left(S_{0-}^{1}, X_{3}^{1}\right)= & 0.3836, \quad \xi^{-}\left(S_{0-}^{2}, X_{3}^{2}\right)=0.3333 \\
& \xi^{-}\left(S_{0-}^{3}, X_{3}^{3}\right)=0.4276 .
\end{aligned}
$$

Step 4. According to (16), we calculate the three-dimensional grey interval number relational degree of $i$ th scheme $S_{i}(i=$ $1,2,3)$ to positive/ negative ideal scheme, respectively, are:

$$
\begin{aligned}
& \gamma^{+}\left(S_{0+}, X_{1}\right)=0.6827, \\
& \gamma^{+}\left(S_{0+}, X_{2}\right)=0.7159, \\
& \gamma^{+}\left(S_{0+}, X_{3}\right)=0.8428 \\
& \gamma^{-}\left(S_{0-}, X_{1}\right)=0.5686, \\
& \gamma^{-}\left(S_{0-}, X_{2}\right)=0.4125, \\
& \gamma^{-}\left(S_{0-}, X_{3}\right)=0.3815 .
\end{aligned}
$$

Step 5. According to (18), we calculate the affiliate value $\mu_{i}(i=1,2,3)$ of $i$ th scheme $S_{i}$ to positive ideal scheme: $\mu_{1}=0.5904, \mu_{2}=0.7508, \mu_{3}=0.8299$.

Step 6. According to the magnitude of $\mu_{i}(i=1,2,3)$, we should arrange the schemes in sequence and select the best.

The bigger the affiliate value $\mu_{i}$ is, the closer the $i$ th scheme and the ideal optimized scheme $S^{+}$are, and the better the decision scheme $S_{i}$ is. Then we could get the result as $\mu_{3}>\mu_{2}>\mu_{1}$. In terms of the above steps, we could get the preferred sequence as $S_{3}>S_{2}>S_{1}$. The primary investment subject is $S_{3}$, followed by $S_{2}$ and $S_{1}$ respectively. In fact, compared to the traditional MCDM problems, the superiority of our proposed method lies in the way of measuring merits of alternatives. The traditional MCDM model measures the merit of alternatives by distance formula. And the method proposed in the paper measures the merit of alternatives by grey relational formula in which all the factors could be included.

\section{Conclusions and Future Work}

This paper takes elements in a system as the points in the $m$-dimensional linear space and regards the observation data of each element at different time and objects as the coordinates of point. Then a three-dimensional grey interval relation degree model based on time, index, and scheme is constructed according to the presented method in the paper. And the method is applied in the interval number dynamic multiattribute index decision making in an investment scheme selection. The method presented in the paper 
makes the traditional decision method simplify a lot and could deal with some uncertain problems. At the same time, we can further study the determination method of index weight and hybrid dynamic decision problem on the basis of this paper. As for the uncertain interval number decision problem, we can also use the fuzzy interval method to resolve it. However, the affiliate function of each scheme must be given previously, so this will bring some difficulties to the uncertain decision making in practical applications. Some comparisons between the traditional MCDM model and the model presented in this paper will be carried out in future research.

\section{Conflict of Interests}

The authors declare that there is no conflict of interests regarding the publication of this paper.

\section{Acknowledgment}

This paper is partially supported by National Project for Education Science Planning (EFA110351).

\section{References}

[1] J. L. Deng, A Text Book of Grey System Theory, Huazhong University of Science and Technology Press, Wuhan, China, 2002.

[2] H. Zhan, Q. Fang, and W. Chen, "Grey correlation method in the decision of bridge design plans," Kybernetes, vol. 38, no. 10, pp. 1812-1818, 2009.

[3] G.-W. Wei, "Gray relational analysis method for intuitionistic fuzzy multiple attribute decision making," Expert Systems with Applications, vol. 38, no. 9, pp. 11671-11677, 2011.

[4] Z. P. Fan and S. H. Xiao, "A relation grade approach for the dynamic multiple attribute decision making," Systems Engineering, vol. 13, no. 1, pp. 23-27, 1995.

[5] Y. M. Yang, "A new relation degree analysis method for dynamic multiple attribute decision making," Systems Engineering and Electronics, vol. 15, no. 11, pp. 9-11, 1997.

[6] W. Y. Zeng and C. Z. Luo, "Comprehensive decision model of interval number," Systems Engineering-Theory \& Practice, vol. 17, no. 11, pp. 48-50, 1997.

[7] J.-Q. Wang, "Research on decision making model of the dynamic multi-attribute system for rewarding good and punishing bad," Systems Engineering and Electronics, vol. 24, no. 3, p. 39, 2002.

[8] Y. G. Dang, S. F. Liu, and B. Liu, "Study on grey incidence decision model of the dynamic multiple-attribute," Engineering Science, vol. 7, no. 2, pp. 69-72, 2005.

[9] W. Li and F. Ye, "Interval appraise method based on grey relation," Systems Engineering and Electronics, vol. 23, no. 2, pp. 55-57, 2001.

[10] J.-J. Zhang, "Method of grey related analysis to multiple attribute decision making problem with interval numbers," Systems Engineering and Electronics, vol. 27, no. 6, pp. 1030-1033, 2005.

[11] Y.-H. Lin, P.-C. Lee, and H.-I. Ting, "Dynamic multi-attribute decision making model with grey number evaluations," Expert Systems with Applications, vol. 35, no. 4, pp. 1638-1644, 2008.
[12] J. J. Zhu and K. W. Hipel, "Multiple stages grey target decision making method with incomplete weight based on multigranularity linguistic label," Information Sciences, vol. 212, pp. 15-32, 2012.

[13] X. X. Chen, "Hybrid grey multiple attribute decision-making method with partial weight information," Kybernetes, vol. 41, no. 5-6, pp. 611-621, 2012. 


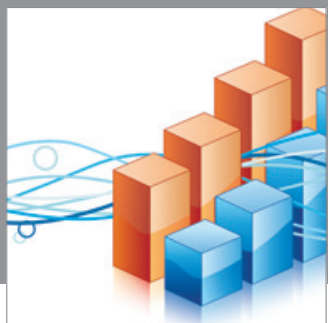

Advances in

Operations Research

mansans

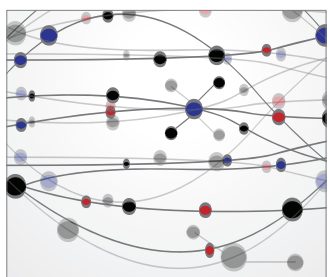

The Scientific World Journal
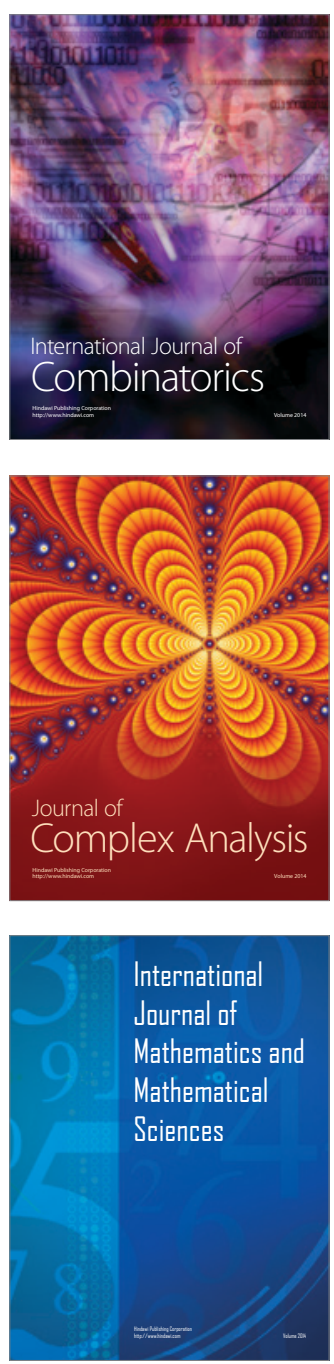
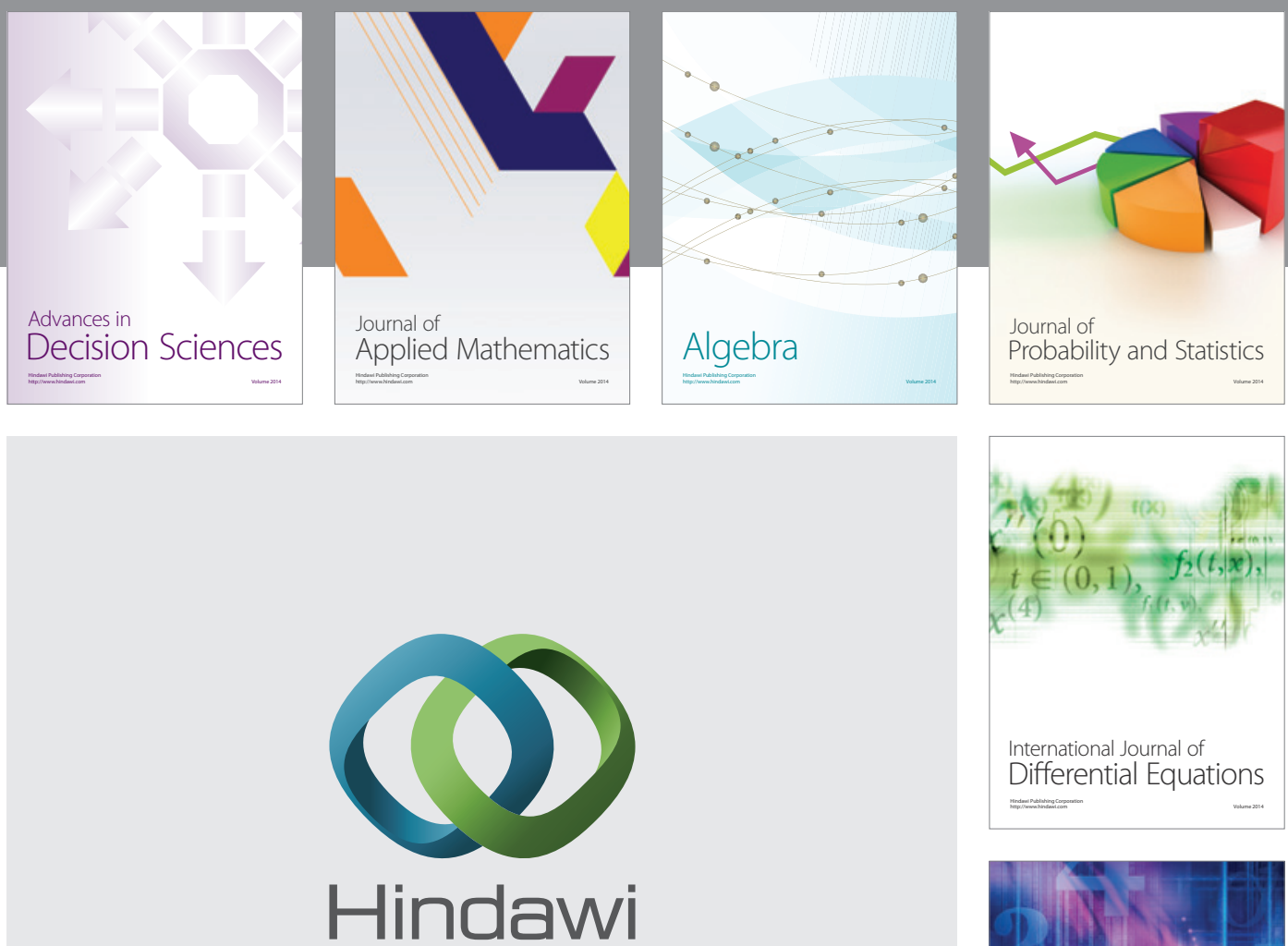

Submit your manuscripts at http://www.hindawi.com
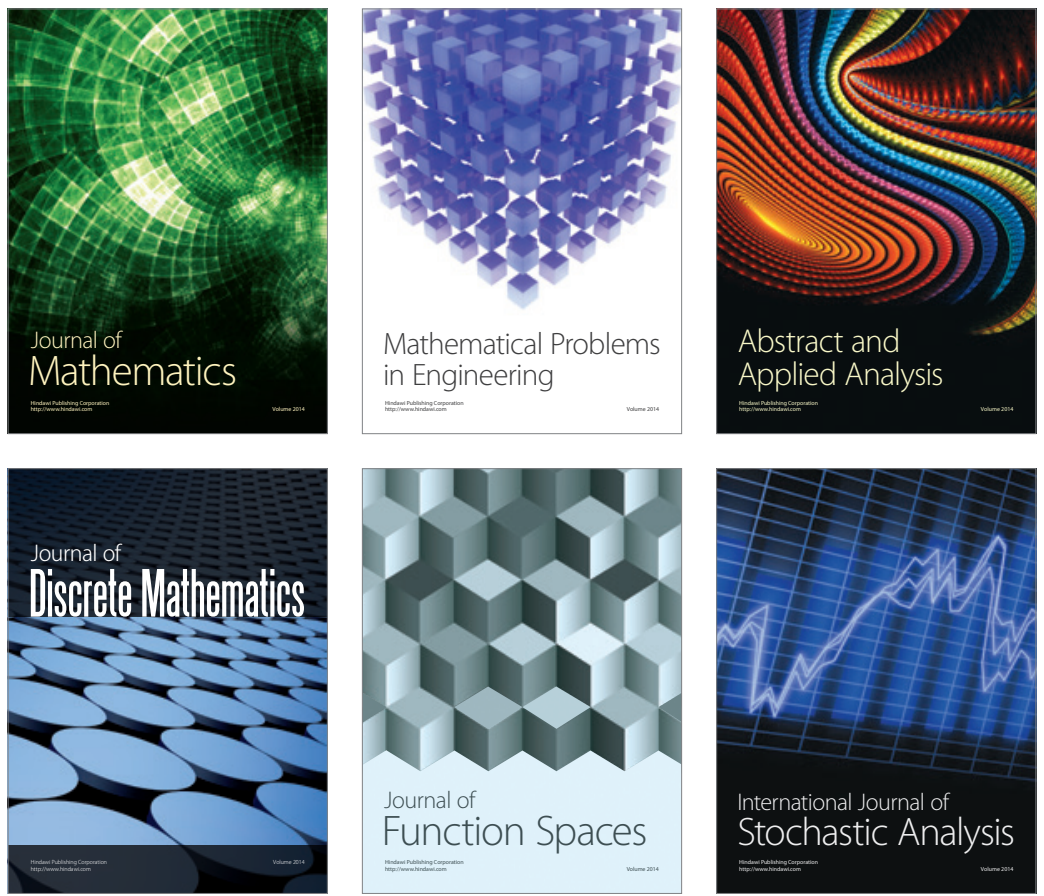

Journal of

Function Spaces

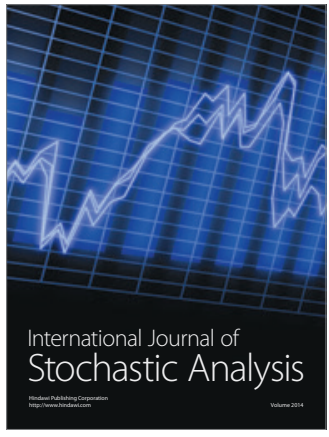

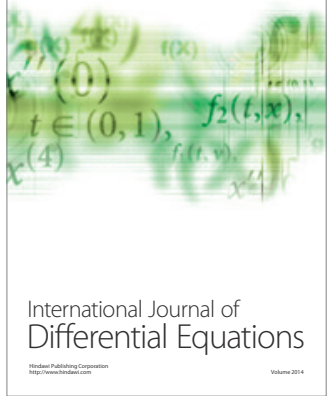
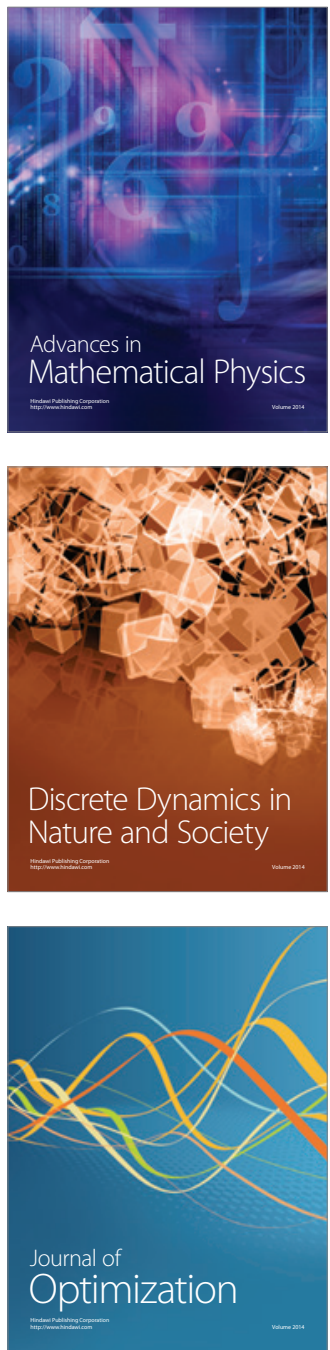